\title{
Über Nitrophenylbiguanide
}

von

\section{Richard Hermann.}

Aus dem Laboratorium für allgemeine Chemie an der k. k. Technischen Hochschule in Graz.

(Vorgelegt in der Sitzung am 8. Juni 1905.)

In Fortsetzung der Untersuchungen über die Biguanide, zu deren Kenntnis das hiesige Laboratorium wiederholt Beiträge geliefert hat, übertrug mir Herr Prof. Emich die Aufgabe, wenn möglich nitrierte Derivate des Phenylbiguanids darzustellen; denselben kommt vielleicht insofern ein gewisses Interesse $\mathrm{zu}$, als nitrierte Biguanide bis nun überhaupt noch nicht bekannt sind. Zur Lösung der Aufgabe bieten die bisberigen Erfahrungen zwei Wege:

Entweder kann der synthetische Weg eingeschlagen werden, indem die bekannte Herth'sche Reaktion ${ }^{1}$ auf nitrierte Aniline in Anwendung gebracht wird; oder kann das fertige Phenylbiguanid dem Nitrierungsprozesse unterworfen werden, in ähnlicher Art, wie J. Thiele ${ }^{2}$ Guanidin durch Einwirkung des Nitrierungsgemisches in seine Nitroverbindung überführte.

Die zu erwartenden Reaktionen würden den Schemen entsprechen:
I. $\mathrm{C}_{6} \mathrm{H}_{4}<\underset{\mathrm{NH}_{2}}{\mathrm{NO}_{2}}+\mathrm{C}_{2} \mathrm{H}_{4} \mathrm{~N}_{4}=\mathrm{C}_{2} \mathrm{H}_{6} \mathrm{~N}_{5}\left(\mathrm{C}_{6} \mathrm{H}_{4}, \mathrm{NO}_{2}\right)$
II. $\mathrm{C}_{2} \mathrm{H}_{6} \mathrm{~N}_{5} \cdot \mathrm{C}_{6} \mathrm{H}_{5}+\mathrm{HNO}_{\mathrm{g}}=\mathrm{C}_{2} \mathrm{H}_{6} \mathrm{~N}_{5}\left(\mathrm{C}_{6} \mathrm{H}_{4} \cdot \mathrm{NO}_{2}\right)+\mathrm{H}_{2} \mathrm{O}$.

1 Monatshefte für Chemie, I, 88 (1880) und IX, 228 (1888); X, 87 (1889),

2. Annalen, 270, 1 (1892). 
Die in dieser Richtung angestellten Versuche haben ergeben, daß der synthetische Weg (I) nicht gangbar ist. Versuche, Dicyandiamid mit nitrierten Anilinen in Reaktion zu bringen, ergaben niemals positive Resultate, vielmehr konnten die Nitroaniline stets unverändert rüıkgewonnen werden. Diese Tatsache erinnert an die Erscheinungen, welche $\mathrm{A}$. Kämpf ${ }^{1}$ bei der Einwirkung von Cyanamid auf Nitroaniline beobachtet hat und es dürften die Gründe, welche er zur Erklärung dieser Anomalien vorführt, auch in unserem Falle Berechtigung finden.

Vor kurzer Zeit fanden Lumière und Perrin , ${ }^{2}$ daß die im Kern hydroxylierten, beziehungsweise methoxylierten primären aromatischen Amine beim Verschmelzen mit Dicyandiamid (Reaktion nach Herth-Smolka) hingegen substituierte Biguanide ergaben und es scheint damit erwiesen, daß für die Bildung von Amidinen die allenfalls vorhandene, stärker oder minder starke elektronegative Gruppe von Einfluß ist.

Zur Darstellung von Nitrophenylbiguaniden verblieb demnach noch der zweite Weg (II), nach welchem es nach Überwindung einiger anfänglichen Schwierigkeiten in der Tat gelang, in das Phenylbiguanidmolekül sukzessive drei Nitrogruppen einzuführen.

Um die Konstitution der gewonnenen Körper festzustellen, wurden sie hydrolytisch gespalten. Hiebei wurden aus den Nitroverbindungen korrespondierend $p$-Nitranilin, (2-4)-Dinitranilin und (2-4-6)-Trinitranilin erhalten, entsprechend den Schemen:

1. $\mathrm{C}_{8} \mathrm{~N}_{6} \mathrm{H}_{10} \mathrm{O}_{2}+4 \mathrm{H}_{2} \mathrm{O}=p-\mathrm{C}_{6} \mathrm{H}_{4} \cdot \mathrm{NO}_{2} \cdot \mathrm{NH}_{2}+2 \mathrm{CO}_{2}+4 \mathrm{NH}_{3}$ 2. $\mathrm{C}_{8} \mathrm{~N}_{7} \mathrm{H}_{9} \mathrm{O}_{4}+4 \mathrm{H}_{2} \mathrm{O}=(2-4)-\mathrm{C}_{6} \mathrm{H}_{3} .\left(\mathrm{NO}_{2}\right)_{2} \mathrm{NH}_{2}+2 \mathrm{CO}_{2}+4 \mathrm{NH}_{3}$ 3. $\mathrm{C}_{8} \mathrm{~N}_{8} \mathrm{H}_{3} \mathrm{O}_{6}+4 \mathrm{H}_{2} \mathrm{O}=(2-4-6)-\mathrm{C}_{6} \mathrm{H}_{2} \cdot\left(\mathrm{NO}_{2}\right)_{3} \mathrm{NH}_{2}+2 \mathrm{CO}_{2}+4 \mathrm{NH}_{3}$.

Die Nitroaniline wurden durch ihren Schmelzpunkt und ihre sonstigen Eigenschaften sowie durch Überführung in die entsprechenden Amidoverbindungen identifiziert, die wieder

1 Berl. Ber., XXXVII, 1681, 1894

2 Chem. Zentralb1., 1905, I. Bd., 730. 
durch ihre spezifischen Farbreaktionen als solche unzweideutig erkannt wurden.

Die Molekulargewichtsbestimmungen ließen auf das einfache Molekül schließen.

Demnach kommen den (kristallwasserfreien) Nitrophenylbiguaniden nachstehende Konstitutionsformeln $z u$ :

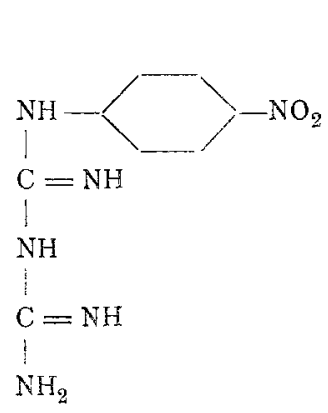

I.

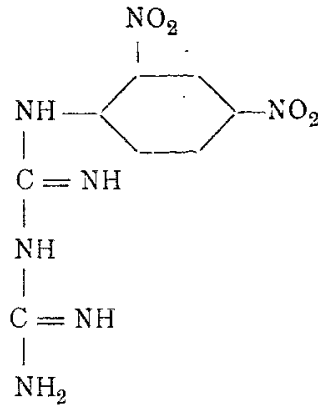

II.

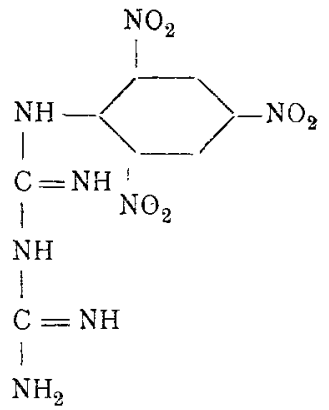

III.

Es wurde demnach der experimentelle Beweis erbracht, daß unter den gewählten Bedingungen die Nitrierung im aromatischen Kerne, erst in der $p$-Stellung zur substituierten Amidogruppe erfolgt und daß jede neu eintretende, den Wasserstoff des Benzols ersetzende Nitrogruppe gegenüber der vorhandenen die Metastellung einnimmt.

Diese Konstitutionsverhältnisse sind analog den bei einfach nitrierten Säuren ${ }^{1}$ oder jenen anderen Ortho- und Parabenzolderivaten, wo jede neu eintretende Nitrogruppe sich in die Metastellung zur bereits vorhandenen stellt.

Dem chemischen Charakter nach stellt der Mononitrokörper eine starke Base dar, welche ein Molekül Kristallwasser bindet und gut kristallisierende Salze mit einem Äquivalent Säure liefert; Dinitrophenylbiguanid wird durch Kalilauge in wässeriger oder alkoholischer Lösung bereits verändert, d. h. tiefrot gefärbt; erst im Trinitrokörper ist der basische Charakter des Biguanids nahezu verschwunden; seine Salze mit Säuren werden schon durch Wasser zersetzt; es zeigt schon schwach

1 A. Hübner, Annalen, 222, 67. 
saure Eigenschaften, 10̈st sich leicht in Lauge und wird aus dieser Lösung beim Verdünnen nicht wieder abgeschieden.

Alle dargestellten Nitrokörper sind trotz der Anhäufung von Stickstoff im Molekül nicht explosiv; beim Erhitzen am Platinblech brennen sie gleichmäßig unter Ausstoßung grünlichweißer Dämpfe und Abscheidung von schwer verbrennbarem Kohlenstoff.

Wie zu erwarten besitzen diese Nitroverbindungen Farbstoffcharakter, nicht alle jedoch ausgesprochenes Färbevermögen. Die Fähigkeit, sich mit oder ohne Hilfe von Beizen auf der Faser zu fixieren, kommt am meisten dem Trinitrokörper $\mathrm{zu}$, der eine tiefrote und besonders lichtechte Farbennuance $z u$ erzeugen im stande ist.

Die Nitrogruppe zeigt sich auch hier als bathochrom, ${ }^{1}$ da sie eine Vertiefung im Farbenton mit sich bringt.

Die Fähigkeit, Kupferverbindungen zu bilden, mangelt allen Nitrophenylbiguaniden; da das nicht nitrierte Phenylbiguanid, gleichwie seine Amidoverbindung, diese nach $\mathrm{H}$. Schiff ${ }^{2}$ an bestimmte Komplexe (in unserem Falle $\mathrm{C}=\mathrm{NH}$ ) gebundene $\mathrm{NH}_{2}$

Reaktion gibt, so kann für ihr Ausbleiben bei den Nitroverbindungen (nicht eine sterische Behinderung, sondern nur) die Gegenwart der stark elektronegativen Nitrogruppe als Ursache angesehen werden.

Anhangsweise soll erwähnt werden, daß durch elektrochemische Reduktion aus der Mononitroverbindung Amidophenylbiguanid isoliert wurde, für dessen Strukturformel sein Spaltungsprodukt sowie die Analyse spricht.

Das Studium dieser und anderer Amidopheny]biguanide, die bei der elektrolytischen Reduktion entstehen, nach verschiedenen Richtungen fortzusetzen, soll das Ziel einer weiteren Arbeit sein.

1 Schütze, Zeitschrift für phys. Chomie, IX, 114 (1892).

2 Annalen, 299, 236 (1898). 


\section{Experimenteller Teil.}

Das $\mathrm{zu}$ den Versuchen benötigte Dicyandiamid wurde größtenteils aus Cyanamidnatrium gewonnen, ${ }^{1}$ wobei sich folgendes Verfahren bewährte:

\section{Darstellung von Dicyandiamid aus Cyanamidnatrium.}

In einem Literkolben, mit passendem Rückflußkühler versehen, wurden $150 \mathrm{~g}$ Ammonsulfat mit wenig Wasser zu einer dicken breigen Masse verrührt und am Wasserbad erhitzt. Hierauf wurden $100 \mathrm{~g}$ fein gepulvertes Cyanamidnatrium in kleinen Portionen unter stetem Umschütteln zugesetzt. Bald zeigte sich die Abscheidung von gelbem öligen Cyanamid, welches sich nach etwa einstündigem Erwärmen und öfterem Unschütteln des Kolbeninhaltes zu Dicyandiamid polymerisierte, wobei das Ende der Reaktion durch Prüfung mit Silbernitrat leicht $\mathrm{zu}$ erkennen war. Nun wurde die breiige Masse mit 80 prozentigem Alkohol im Soxhletapparat ausgelaugt und das gewonnene Dicyandiamid nötigenfalls aus Wasser umkristallisiert.

Die Ausbeute betrug 80 bis $90 \%$ der theoretisch möglichen; es ist dabei dem Umstande Rechnung getragen, daß das Ausgangsmaterial, wie durch die Analysen erwiesen ist, etwa $80 \%$ reines Cyanamidnatrium enthielt.

Aus dem Dicyandiamid wurde nach der Methode von Smolka-Friedreich ${ }^{2}$ Phenylbiguanidchlorhydrat dargestellt.

Einwirkung von rauehender Salpetersäure auf Phenylbiguanid.

Die substituierten Biguanide sind als relativ wasserstoffarme Körper leicht nitrierbar; doch entstehen bei der Einwirkung von rauchender Salpetersäure auf eine sirupartige, schwefelsaure Lösung von Phenylbiguanid außer den drei

1 Ein Teil wurde dem Laborntorium von der Deutschen Gold- und Siberscheideanstalt vormals Rößler, Frankfurt a. M., zur Verï̈gung gestellt, wofür auch an dieser Stelle bestens gedankt sei. F, Emich.

2 Monatshefte für Chemie, IX., 228 (1888). 
Nitroverbindungen noch andere Nebenprodukte in geringer Menge; ihre Trennung von den Nitroköipern wie auch die Isolierung dieser bereitete im Anfange der Untersuchung einige Schwierigkeit. Erst später stellte sich heraus (und damit hat diese Trennungsmethode völlig an Interesse verloren), daß bei geeigneter Nitrierung ausschließlich das Mononitroderivat gebildet wird, das nach und nach in die $\mathrm{Di}$ - und Trinitroverbindung übergeführt werden kann.

\section{Mononitrophenylbiguanid.}

Darstellung des Sulfats. $10 \mathrm{~g}$ Phenylbiguanidchlorhydrat wurden mit $18 \mathrm{~cm}^{3}$ konzentrierter Schwefelsäure bis zur vollständigen Lösung verrieben und der sirupartigen Masse $2.5 \mathrm{~cm}^{3}$ rauchende Salpetersäure (spezifisches Gewicht 1.51) in kleinen Portionen unter stetem Rühren zugesetzt, wobei die Temperatur 30 bis $40^{\circ}$ betrug. Wurde die braungrüne Flüssigkeit hierauf in Wasser gegossen, so fiel beim unvollständigen Neutralisieren mit Lauge der Nitrokörper aus, der nach dem Auswaschen einige Male aus heißem Wasser, nötigenfalls unter Zusatz von einer Spur Schwefelsäure, umkristallisiert wurde.

Die Analysen der bei $105^{\circ}$ getrockneten Substanz ergaben folgende Resultate:

$0.7039 \mathrm{~g}$ Substanz gaben $0.3025 \mathrm{~g} \mathrm{Ba} \mathrm{SO}_{4}$.

In 100 Teilen:

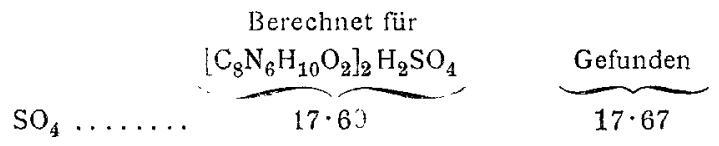

$0.1872 \mathrm{~s}$ Substanz, mit Bleichromat verbrannt, gaben $52.0 \mathrm{~cm}^{3}$ feuchten Stickstoff bei $16^{\circ}$ und $729 \mathrm{~mm}$ Barometerstand.

In 100 Teilen:

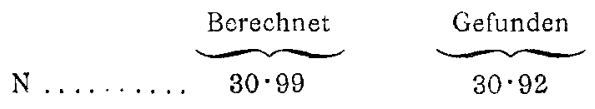

Demnach ist der gefundene Körper das neutrale Sulfat des Mononitrophenylbiguanids und es kommt ihm die empirische Formel zu:

$$
\left[\mathrm{C}_{8} \mathrm{~N}_{6} \mathrm{H}_{10} \mathrm{O}_{2}\right]_{2} \mathrm{H}_{2} \mathrm{SO}_{4}
$$


Das Sulfat stellt weiße, haarförmige, oft verfilzte Nädelchen dar, oft fein verästelte Gebilde. Es ist in heißem Wasser wenig löslich, leichter auf Zusatz von etwas Säure. Aus der sauren Lösung wird die Verbindung durch Basen wieder ausgeschieden. Die Substanz schmilzt unscharf bei $254^{\circ}$ und reagiert auf Lackmuspapier neutral.

Aus der Mutterlauge des Sulfats fiel die freie Base bei weiterem Zusatz von starker Lauge (unter guter Kühlung) als eigelber Körper aus, der nach dem Auswaschen mit Wasser aus kochendem Alkohol umkristallisiert wurde.

Kristallwasserbestimmung:

$1.123 \mathrm{~g}$ exsikkatortrockene Substanz verloren, bei $105^{\circ}$ getrocknet, $0.087+\mathrm{g}$

$\mathrm{H}_{2} \mathrm{O}$, entsprechend $7 \cdot 78 \%$ Wasser, gegen $7 \cdot 50 \%$ der Theorie (für ein Molekül Kristallwasser gerechnet).

Die Analyse der bei $105^{\circ}$ getrockneten Substanz lieferte folgende Zahlen:

$0.1104 \mathrm{~g}$ Substanz gaben $38.0 \mathrm{~cm}^{3}$ feuchten $\mathrm{N}$ bei $21^{\circ}$ und $732 \mathrm{~mm}$ Druck.

In 100 Teilen:

N $\ldots \ldots \ldots \ldots \underbrace{\begin{array}{c}\text { Berechnct für } \\ \mathrm{C}_{8} \mathrm{~N}_{6} \mathrm{H}_{10} \mathrm{O}_{2}\end{array}}_{37 \cdot 88} \quad \underbrace{\text { Gefunden }}_{37 \cdot 96}$

Demnach kommt der exsikkatortrockenen Substanz die empirische Formel zu:

$$
\mathrm{C}_{8} \mathrm{~N}_{6} \mathrm{H}_{10} \mathrm{O}_{2}+\mathrm{H}_{2} \mathrm{O} \text {. }
$$

Die Molekulargewichtsbestimmung ergab nach der Beckmannschen Siedemethode folgende Resultate:

\begin{tabular}{|c|c|c|c|c|}
\hline \multirow{2}{*}{ Substanz } & \multirow{2}{*}{ Erhöhung } & \multirow{2}{*}{$\begin{array}{c}\text { Kon- } \\
\text { zentration }\end{array}$} & Gefundenes & Berechnetes \\
\hline & & & \multicolumn{2}{|c|}{ Molekulargewicht } \\
\hline \multicolumn{5}{|c|}{$23.46 \mathrm{~g}$ Alkohol, Siedepunkt $79 \cdot 9^{\circ}, K=11 \cdot 5$} \\
\hline $0 \cdot 1742 g$ & 0.036 & 0.742 & 237 & 222 \\
\hline \multicolumn{5}{|c|}{$27 \cdot 25 g$ Alkohol, Siedepunkt $79 \cdot 9^{\circ}, K=11 \cdot 5$} \\
\hline $0.1330 \mathrm{~g}$ & 0.026 & $0 \cdot 488$ & 216 & 222 \\
\hline $0.2531 \mathrm{~g}$ & 0.050 & 0.928 & 213 & 222 \\
\hline
\end{tabular}


In heißem Wasser ist die Base wenig löslich, leicht in Alkohol beim Kochen. Die Kristalle, die im kristallwasserhältigen Zustande eine eigelbe Farbe zeigen, welche beim Trocknen auf $105^{\circ}$ in eine gelborange übergeht, sind als prismatische Blättchen anzusprechen, welche wegen der mikrokristallinischen Struktur hinsichtlich ihrer Kristallform nicht näher bestimmt werden konnten; im Polarisationsapparat erwiesen sie sich als doppeltbrechend. ${ }^{1}$ Die Substanz schmilzt bei $182^{\circ} \mathrm{zu}$ einer dunkelroten Flüssigkeit; ihre Reaktion auf Lackmus ist basisch. In kalter konzentrierter Kalilauge ist die Base auch beim längeren Stehen kaum löslich, was mit dem stark basischen Charakter zusammenhängen dürfte. Die alkoholische Lösung gibt mit Pikrinsäure einen gelben, scheinbar amorphen Niederschlag, der in der Wärme leicht löslich ist und beim Erkalten flockig ausfällt. Mit Sibernitrat ensteht ein hellgelber, flockiger Niederschlag, leicht löslich in Salpetersäure.

Spaltungsversuch. $2 \mathrm{~g}$ Mononitrophenylbiguanidsulfat wurden mit $100 \mathrm{~cm}^{3}$ starker Kalilauge aufgeschlemmt und in einer Stahlbombe bei einer Temperatur von 125 bis $130^{\circ}$ durch drei Stunden hindurch belassen. Die von den bläulich schimmernden, gelben Kristallen gesonderte Lösung wurde wiederholt mit Äther ausgeschüttelt; nach Vereinigung mit den zuerst erhaltenen Kristallen wurde ein Produkt gewonnen, aus dem sich nach der bekannten Methode ${ }^{2}$ weder Ortho- noch Metanitranilin isolieren ließ. Es zeigte nach dem Umkristallisieren aus Benzol den Schmelzpunkt von $147^{\circ}$ (Schmelzpunkt für $p$-Nitranilin $147^{\circ}$ ). Zur Amidoverbindung reduziert, ergab diese die für $p$-Diamine charakteristische Lauth'sche Farbreaktion, desgleichen die Indamin- und Indophenolreaktion.

Die mit Baryumhydroxyd wie mit verdünnter Schwefelsäure durchgeführten Spaltungsversuche führten zu denselben Resultaten.

1 Herrn Prof. J. Rumpf, der die dargestellten Nitroverbindungen in kristallographischer Hinsicht zu beurteilen die Güte hatte, sei auch an dieser Stelle der Dank hiefür ausgesprochen.

2 Annalen, 208, 300 (1881). 
Bei der Einwirkung von Mononitrophenylbiguanidsulfat auf Kalilauge bei höherer Temperatur findet also eine Abspaltung von $p$-Nitranilin statt und es tritt demnach bei der Nitrierung des Phenylbiguanids die Nitrogruppe in den aromatischen Kern, und $z w a r$ in die Parastellung zur Amidogruppe.

\section{Dinitrophenylbiguanid.}

Darstellung des Sulfats. $10 \mathrm{~g}$ Mononitrophenylbiguanidsulfat wurden mit wenig konzentrierter Schwefelsäure bis zur völligen Lösung verrieben und $3 \mathrm{~cm}^{3}$ rauchende Salpetersäure (spezifisches Gewicht 1.51) in kleinen Portionen und stetem Rühren eingetragen. Die Temperatur wurde einige Zeit auf 75 bis $80^{\circ}$ gehalten, wobei sich das Reaktionsgemisch tiefgelb färbte. Die Nitrierung konnte als beendet angesehen werden, wenn eine herausgenommene Probe, in ammoniakalisches Wasser gegossen, orangegelbe, seidenglänzende Nädelchen abschied, die sich darin teilweise mit blutroter Farbe lösten; das Nitriergemisch wurde alsdann in Wasser gegossen und der ausgefallene Nitrokörper einige Male aus kochendem schwefelsäurehaltigen Wasser umkristallisiert.

Selbstredend ist zur Darstellung des Dinitrokörpers eine nur intermediäre Bildung des Mononitrokörpers nötig, so daß dieser weiterhin nicht besonders isoliert $z u$ werden braucht.

Die Analysen lieferten folgende Zahlen:

$0.7408 \mathrm{~g}$ Substanz gaben $0.2674 \mathrm{~g} \mathrm{BaSO}_{4}$.

In 100 Teilen:

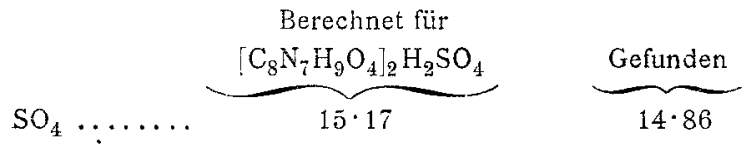

$0 \cdot 1620 \mathrm{~g}$ Substanz, mit Bleichromat verbrannt, gaben $45 \cdot 4 \mathrm{~cm}^{3}$ feuchten $\mathrm{N}$, bei $20^{\circ}$ und $733 \mathrm{~mm}$ Barometerstand.

In 100 Teilen:

$$
\text { N ........ } \underbrace{\text { Berechnet }}_{31 \cdot 01} \quad \underbrace{\text { Gefunden }}_{31 \cdot 11}
$$


Demnach ist der gefundene Körper das neutrale Sulfat des Dinitrophenylbiguanids und kommt ihm die empirische Formel zu:

$$
\left[\mathrm{C}_{8} \mathrm{~N}_{7} \mathrm{H}_{9} \mathrm{O}_{4}\right]_{2} \mathrm{H}_{2} \mathrm{SO}_{4} \text {. }
$$

Der Dinitrokörper zeigt unterm Mikroskop hellgelbe, breite Nadeln, die häufig übereinander gelagert sind. Sein Schmelzpunkt liegt bei $234^{\circ}$; er reagiert auf Lackmus neutral.

Die freie Base fiel auf Zusatz von Ammoniak zu der wässerigen Lösung des Sulfats als orangegelbe, verfilzte Kristallmasse in der Kälte aus; sie wurde mehrmals aus viel heißem Wasser umkristallisiert.

Die Analysen der bei $105^{\circ}$ getrockneten Substanz lieferten folgende Zahlen:

$0.2203 \mathrm{~g}$ Substanz gaben $74.8 \mathrm{~cm}^{\mathrm{a}}$ feuchten $\mathrm{N}$ bei $24^{\circ}$ und $733 \mathrm{~mm}$ Barometerstand.

In 100 Teilen:

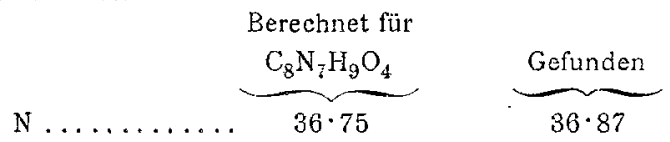

$0.2096 \mathrm{~g}$ Substanz, mit $\mathrm{Pb} \mathrm{CrO}_{1}+\mathrm{K}_{2} \mathrm{Cr}_{2} \mathrm{O}_{7}$ verbrannt, gaben $0.2753 g \mathrm{CO}_{2}$ und $0.066 \mathrm{~g} \mathrm{H}_{2} \mathrm{O}$.

$0.2198 g$ Substanz gaben $0.0734 g \mathrm{H}_{2} \mathrm{O}$.

In 100 Teilen:

$\begin{array}{ll}\text { C } \ldots \ldots \ldots & \begin{array}{l}\text { Berechnet } \\ \text { H } \ldots \ldots \ldots \ldots\end{array} \quad 3.92\end{array} \quad \underbrace{\left\{\begin{array}{l}3.52 \\ 3.71\end{array}\right.}_{\begin{array}{l}35.81 \\ \text { Gefunden }\end{array}}$

Die Molekulargewichtsbestimmung, nach der Beckmann'schen Siedemethode ausgeführt, ergab folgende Resultate:

\begin{tabular}{|c|c|c|c|c|}
\hline Sulistanz & Erhöhung & $\begin{array}{c}\text { Kon- } \\
\text { zentration }\end{array}$ & Gefundenes & Berechnetes \\
\hline \multicolumn{2}{|c|}{$\mathbf{2 7 \cdot 2 5} g$ Alkohol, Siedepunkt $79 \cdot 9^{\circ}, K=11 \cdot 5$} \\
\hline $0.1604 g$ & 0.024 & 0.588 & 282 & 267 \\
$0.0860 g$ & 0.013 & 0.315 & 278 & 267 \\
\end{tabular}


Es kommt demnach der Base die empirische Formel zu:

$$
\mathrm{C}_{8} \mathrm{~N}_{7} \mathrm{H}_{9} \mathrm{O}_{4}
$$

Die Base stellt orangegelbe, nadelförmige Kriställchen dar, die sich im Polarisationsapparat als doppeltbrechend erwiesen; sie sind leicht in Alkohol und Ather sowie in ammoniakalischem heißen Wasser löslich.

Die orangegelbe alkoholische Lösung geht auf Zusatz von einigen Tropfen Lauge in eine tiefrote Flüssigkeit über. Bei $193^{\circ}$ schmilzt der Dinitrokörper zu einer dunkelroten Flüssigkeit; auf Lackmus reagiert er schwach basisch.

Aus der alkoholischen Lösung der Base wird auf Zusatz von konzentrierter Oxalsäure das hellgelbe Oxalat gefällt; breite Nädelchen, oft drusig angeordnet, in Wasser, Alkohol und Äther kaum löslich.

Mit Pikrinsäure fällt ein hellgelber Niederschlag aus, der aus büscheligen Aggregaten besteht und leicht löslich in heißem Wasser ist; auf Zusatz von Silbernitrat zeigt sich ein gelber, scheinbar amorpher Niederschlag, der, in der Wärme unlöslich, leicht von Salpetersäure gelöst wird.

Spaltungsversuch. $2 g$ Dinitrophenylbiguanidsulfat wurden mit verdünnter Schwefelsäure $(40: 100)$ in der Bombe bei einer Temperatur von 160 bis $170^{\circ}$ durch 3 Stunden erhitzt; nach dem Eingießen des Rohrinhaltes in Wasser wurde ein hellgelber kristallinischer Niederschlag erhalten, der nach mehrmaligem Umkristallisieren aus Alkohol den Schmelzpunkt von 181 bis $182^{\circ}$ zeigte.

Das nach der Angabe von Rudnew ${ }^{1}$ aus seiner Acetylverbindung dargestellte (2-4)-Dinitroanilin erwies sich hinsichtlich äußerem Habitus und sonstigen Eigenschaften als ident mit dem erhaltenen Spaltungsprodukte; es zeigte auch nach mehrmaligem Umkristallisieren den konstanten Schmelzpunkt von 180 bis $181^{\circ}$ (Rudnew gibt $175^{\circ}$ an).

Spaltungsprodukt sowie das dargestellte (2-4)-Dinitroanilin $z u$ Triamidobenzol reduziert, ergaben in Anwesenheit

1 Zeitschrift für Chemie, XIV, 202 (1871).

2 Berl. Ber, XII, 1345 (1879). 
oxydierender Agenzien in der Wärme den für (1-2-4)-'Triamidobenzol charakteristischen rotbraunen Farbstoff (Eurhodinreaktion). ${ }^{1}$

Bei der Zersetzung von Dinitrophenylbiguanidsulfat mit verdünnter Schwefelsäure bei höherer Temperatur findet eine Abspaltung von unsymmetrischem Dinitranilin statt und es hat demnach bei weiterer Nitrierung des Mononitrophenylbiguanids die eintretende Nitrogruppe zur vorhandenen die Metastellung eingenommen.

\section{Trinitrophenylbiguanid.}

Darstellung des Sulfats. $10 \mathrm{~g}$ Dinitrophenylbiguanidsulfat wurden mit wenig konzentrierter Schwefelsäure verrieben und $4 \mathrm{~cm}^{3}$ rauchende Salpetersäure (spezifisches Gewicht 1.51) in kleinen Portionen eingetragen; dabei wurde die Temperatur zwischen 105 bis $110^{\circ}$ gehalten. Das Ende der Reaktion konnte an der ins Orange spielenden Färbung des Nitriergemisches erkannt werden; eine Probe, in Wasser gegossen, zeigte an der Oberfläche der Flüssigkeit seidenglänzende, tiefgelbe Schüppchen. Mit schwach ammoniakalischem Wasser aufgekocht, erschien ein roter Körper mit goldigem Oberflächenschimmer. Auf Zusatz von verdünnter Essigsäure blieb er unverändert.

Der aus dem Nitriergemisch gewonnene Nitrokörper wurde aus heißem schwefelsäurehaltigem Wasser mehrmals umkristallisiert, am Filtrierpapier abgepreßt und im Exsikkator getrocknet.

Die Analyse ergab folgende Zahlen:

$0.6550 \mathrm{~g}$ Substanz gaben $0.2085 \mathrm{~g} \mathrm{Ba} \mathrm{SO}_{4}$.

In 100 Teilen:

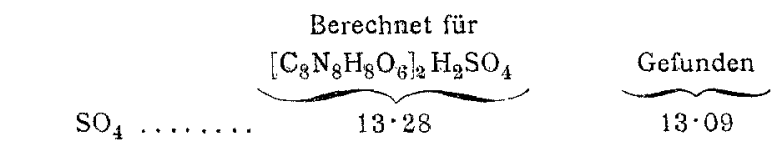

Berl. Ber., XXII, 856 (1889). 
Demnach ist der gefundene Körper d'as neutrale Sutfat des Trinitrophenylbiguanids und kommt ihm die empirische Formel zu:

$$
\left[\mathrm{C}_{8} \mathrm{~N}_{8} \mathrm{H}_{8} \mathrm{O}_{6}\right]_{2} \mathrm{H}_{2} \mathrm{SO}_{4} \text {. }
$$

Der Körper zeigt schon beim Stehen an der Luft Neigung, in die freie Base überzugehen.

Unter dem Mikroskop zeigt das Sulfat breite, unregelmäßige Blättchen von tiefgelber Farbe. Beim Kochen mit Wasser oder Alkohol spaltet es die freie Base vollends ab; es reagiert auf Lackmus sauer; sein Schmelzpunkt liegt über $270^{\circ}$.

Die freie Base fiel aus der Lösung des Sulfats beim Kochen mit einer Spur Ammoniak aus; sie wurde mit heißem Wasser ausgewaschen, bis dieses klar ablief. Bei unvollkommener Nitrierung kann die Base durch Zusatz von verdünnter Essigsäure und nach ihrer Isolierung durch Auslaugen mit Äther und Alkohol im Soxhletapparat frei von beigemengten Verunreinigungen erhalten werden. Zum öfteren genügt hiefür ein Lösen der Base in konzentrierter Essigsäure und nachherige fraktionierte Fällung mit Ammoniak.

Die Analysen der bei $105^{\circ}$ getrockneten Substanz lieferten folgende Zahlen:

$0.1611 \mathrm{~g}$ Substanz, mit $\mathrm{PbCrO}_{4}$ verbrannt, gaben $52.7 \mathrm{~cm}^{3}$ feuchten $\mathrm{N}$ bei $22^{\circ}$ und $739 \mathrm{~mm}$ Barometerstand.

In 100 Teilen:

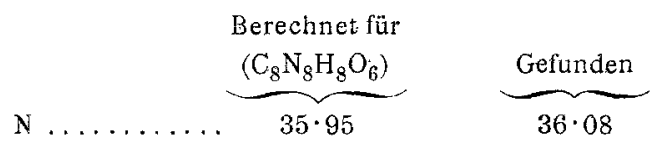

$0.2021 \%$ Substanz, mit $\mathrm{PbCrO} \mathrm{Cr}_{4}+\mathrm{K}_{2} \mathrm{Cr}_{2} \mathrm{O}_{7}$ verbrannt, gaben $0.2265 \mathrm{~g} \mathrm{CO}_{2}$ und. $0.0539 \mathrm{~g} \mathrm{H}_{2} \mathrm{O}$.

In 100 Teilen:

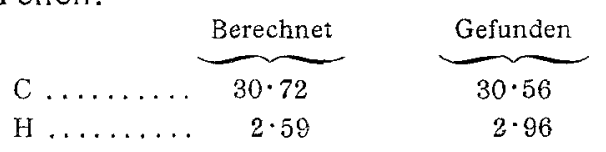

Die Molekulargewichtsbestimmung, nach der Beckmann'schen Siedemethode ausgeführt, ergab folgende Resultate: 


\begin{tabular}{|c|c|c|c|c|}
\hline \multirow{2}{*}{ Substan $z$} & \multirow{2}{*}{ Erhöhung } & \multirow{2}{*}{$\underset{\text { Kontration }}{\text { Kon- }}$} & Gefundenes & Berechnetes \\
\hline & & & \multicolumn{2}{|c|}{ Molekulargewicht } \\
\hline \multicolumn{5}{|c|}{$20 \cdot 70 \mathrm{~g}$ Eisessig, Siedepunkt $118^{\circ}, K=331$} \\
\hline $0.1323 g$ & 0.070 & 0.638 & 301 & 312 \\
\hline $0.2876 g$ & 0.155 & $1 \cdot 389$ & 296 & 312 \\
\hline \multicolumn{5}{|c|}{$37.08 g$ Eisessig, Siedepunkt $118^{\circ}, K=33$} \\
\hline $0.1436 g$ & 0.042 & 0.387 & 304 & 312 \\
\hline $0.1675 \mathrm{~g}$ & 0.050 & 0.451 & 298 & 312 \\
\hline
\end{tabular}

Demnach kommt der Base die empirische Formel zu:

$$
\mathrm{C}_{8} \mathrm{~N}_{8} \mathrm{H}_{8} \mathrm{O}_{6} \text {. }
$$

Die Base ist in Wasser, Alkohol, Äther, Benzol, Petroläther sowie verdünnten Säuren kaum löslich, wenig in Aceton, löslich in konzentrierten Mineralsäuren sowie Eisessig, leichter bei Zusatz einer Spur von Natriumcarbonat; löslich in kochendem Anilin und Phenol. In verdünnten Mineralsäuren bei Gegenwart von Phenol ist die Base leicht löslich, durch Ammoniak wieder fällbar; von starker Lauge wird sie mit orangeroter Farbe aufgenommen.

Die mikroskopischen Kristalle haben den Habitus kurzer Blättchen, die sich scheinbar öfter in Zwillingsstellung befinden und als doppeltbrechend erweisen. Sie zeigen im auffallenden Lichte goldig glänzenden Oberflächenschimmer, im durchfallenden Lichte erscheinen sie rötlichviolett gefärbt.

Die Base ist sehr hygroskopisch; die exsikkatortrockene Substanz gibt beim Trocknen auf $105^{\circ} 2 \cdot 55 \%$ ihres Gewichtes an Wasser ab; bei $239^{\circ}$ (unscharf) schmilzt sie zu einer braunen Flüssigkeit.

1 Die molekulare Siedepunktserhöhung für Eisessig wurde für unseren Fall durch Versuche mit Mononitrophenylbiguanid (Molekulargewicht bekannt) und (2-4-6)-Trinitranilin (Schmelzpunkt $186^{\circ}$ ) festgestellt und nach der Formel $K=\frac{0.02 . T^{2}}{\omega}$ mit rund 33 berechnet. 
Die Spaltung des Trinitrophenylbiguanidsulfats wurde mittels verdünnter Schwefelsäure in der schon oben angegebenen Art bewirkt. Aus der Reaktionsmasse konnte ein kristallinischer, bläulich schimmernder Körper isoliert werden, der, aus Eisessig umkristallisiert, den Schmelzpunkt von $186^{\circ}$ zeigte.

Das aus Pikrylchlorid nach der Vorschrift von Pisan ${ }^{1}$ dargestellte Pikramid, das entgegen seiner Angabe in viel heißem Wasser merklich löslich war, erwies sich mit dem Spaltungsprodukt als ident. Zur weiteren Kennzeichnung wurden beide Körper nach den Angaben Hepp's ${ }^{2}$ in Triamidophenolchlorhydrat übergeführt, welches isoliert gewöhnlichem Leitungswasser eine blaue Farbe erteilte. Somit ist das Spaltungsprodukt von Trinitrophenylbiguanid als (2-4-6)-Trinitroanilin anzusprechen.

Bei der Einwirkung von Trinitrophenylbiguanidsulfat und verdünnter Schwefelsäure bei höherer Temperatur findet eine Abspaltung von (2-4-6)-Trinitroanilin statt und es hat demnach bei weiterer Nitrierung des Dinitrophenylbiguanidsulfats die eintretende Nitrogruppe $z u$ den beiden vorhandenen die Metastellung eingenommen.

\section{Anhang.}

\section{Amidophenylbiguanid.}

Die elektrolytische Reduktion von Mononitrophenylbiguanidsulfat gelang in schwefelsaurer Lösung bei Anwendung einer Bleikathode, wobei anlehnend an die Versuche von Häussermann ${ }^{3}$ folgendermaßen verfahren wurde:

$5 g$ Mononitrophenylbiguanidsulfat wurden mit Schwefelsäure vom spezifischen Gewicht 1.2 und Alkohol in den

1 Compt. rend., XXXIX, 852.

2 Annalen, 215, 350 (1882).

3 Moeller, Die elektrochemische Reduktion der Nitroderivate. Halle a. S., 1893. 
Kathodenraum gebracht (spezifisches Gewicht der Schwefelsäure im Anodenraum 1'1). Die Temperatur war 50 bis $60^{\circ}$; die Konzentration des Gemisches wurde durch einen Rückflußkühler konstant gehalten. Stromstärke 3 bis 3.5 Ampère auf $100 \mathrm{~cm}^{2}$ Kathodenoberfläche, $E=1$ Volt.

Es zeigte sich nach einiger Zeit Rötung der elektrolysierten Flüssigkeit, vielleicht durch intermediäre Bilaung eines Azo. körpers hervorgerufen, später schieden sich feine weiße Kristallnädelchen aus, die aus Alkohol umkristallisiert wurden.

Bei Verwendung einer Zinkkathode in salzsaurer Lösung unter sonst gleichen Bedingungen war ebenfalls eine Kristallabscheidung bemerkbar; die Elektrolyse wurde fortgesetzt, bis Kalilauge keine Gelbfärbung mehr lieferte und die Probe mit $\mathrm{CuSO}_{4}$ und Lauge einen violetten Niederschlag ergab.

Die Analyse ergab folgende Zahlen:

$0.1371 \mathrm{~g}$ Substanz gaben $41.9 \mathrm{~cm}^{5}$ feuchten $\mathrm{N}$ bei $16^{\circ}$ und $740 \mathrm{~mm}$ Barometerstand.

In 100 Teilen:

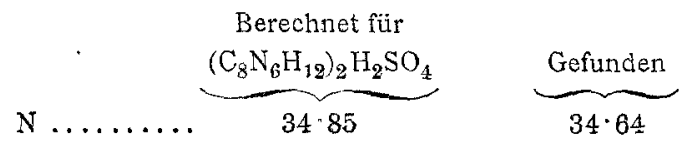

Demnach ist der gefundene Körper das neutrale Sulfat des Amidophenylbiguanids und kommt ihm die empirische Formel zu:

$$
\left(\mathrm{C}_{8} \mathrm{~N}_{6} \mathrm{H}_{12}\right)_{2} \mathrm{H}_{2} \mathrm{SO}_{4} \text {. }
$$

Die Kristalle, weiße breite Nädelchen, öfter prismatische Blättchen, sind in Wasser und Alkohol leicht löslich und geben mit Kupfersulfat und Lauge die aus feinen, fächerförmig angeordneten Nadeln bestehende, rosenrote Kupferverbindung. Auf Zusatz von Goldchlorid zur Amidoverbindung erhält man anscheinend kolloidale Goldlösungen, mit einem Überschuß von Goldchlorid nimmt die Flüssigkeit eine intensiv grüne Färbung an.

Mit ammoniakalischer Silberlösung erhält man in der Wärme einen Silberspiegel. Auf Fehling'sche Lösung reagiert der Amidokörper nicht. 
Spaltungsversuch. Behuís weiterer Aufklärung der Konstitution wurde die Verbindung mit Schwefelsäure (33:100) in der Bombe durch 4 Stunden auf $170^{\circ}$ erhitzt. Die aus der schwefelsauren Lösung mittels Lauge und Äther isolierten Kristalle zeigten den Schmelzpunkt von $144^{\circ}$ (Schmelzpunkt für $p$-Phenylendiamin $144^{\circ}$ ) und gaben die andernorts erwähnten charakteristischen Farbreaktionen auf $p$-Phenylendiamin.

Demnach wird bei der elektrolytischen Reduktion von Mononitrophenylbiguanidsulfat Amidophenylbiguanid gebildet, dem nachstehende Konstitutionsformel zukommt:

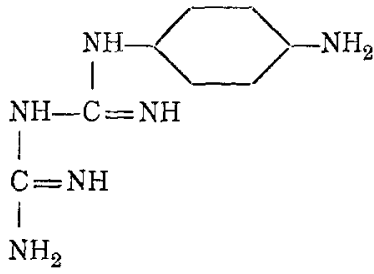

